\title{
Análise do Mecanismo de Incentivo Prefeitura em Locais do Foursquare-Swarm
}

\author{
William de Oliveira Souza ${ }^{1}$, Vinícius F. S. Mota $^{2}$, Thiago H. Silva ${ }^{1}$ \\ ${ }^{1}$ Universidade Tecnológica Federal do Paraná- Curitiba, PR, Brasil \\ ${ }^{2}$ Universidade Federal do Espírito Santo - Vitória, ES, Brazil. \\ william@alunos.utfpr.edu.br, vinicius.mota@inf.ufes.br, thiagoh@utfpr.edu.br
}

\begin{abstract}
This paper presents a study on the mechanism of incentive mayorship used in Foursquare-Swarm. Mayor is the user who performed the most check-ins in the last thirty days in a given location. Through automatic temporal monitoring, we study how alternations and disputes for mayorship occur at the locations. Data were collected from hundreds of locations in Curitiba (Brazil) and Chicago (United States). We identified, for example, that well known American food chains may have a more significant influence on the mayorship dispute in Curitiba. This study provides a better understanding of the mechanism of incentive mayorship, helping to determine where it is advantageous. This can assist in improving user engagement on social web systems.
\end{abstract}

Resumo. Este trabalho apresenta um estudo sobre o mecanismo de incentivo prefeitura utilizado no Foursquare-Swarm. Prefeito é o usuário que mais realizou check-ins nos últimos trinta dias em um determinado local. Através de um monitoramento automático temporal, estudamos como ocorrem as alternâncias e disputa de prefeitos nos locais. Foram coletados dados de centenas de locais em Curitiba (Brasil) e Chicago (Estados Unidos). Identificamos, por exemplo, que grandes redes americanas de alimentação podem influenciar mais significativamente a disputa por prefeitura em Curitiba. Este estudo fornece um melhor entendimento do mecanismo de incentivo prefeitura, ajudando a determinar locais em que o mesmo é vantajoso. Isso pode auxiliar no aprimoramento do engajamento de usuários em sistemas da web social.

\section{Introdução}

Redes sociais baseadas em localização (LBSNs) fornecem serviços baseados em localização física como um dos principais recursos. Dentre as LBSNs, destaca-se o Foursquare-Swarm ${ }^{1}$, que cresceu de 10 milhões de usuários em 2011 [Pontes et al. 2012] para 25 milhões em 2018.

O aplicativo utiliza um tipo de gamificação ${ }^{2}$ denomidado prefeitura, são prefeitos de estabelecimentos os usuários que mais realizaram check-ins ${ }^{3}$ nos últimos 30 dias no local em questão. As prefeituras podem ser disputadas tanto entre amigos quanto entre pessoas desconhecidas. Pelo fato do local estar habilitado para qualquer pessoa realizar check-in através do aplicativo, ser prefeito sugere que o usuário é uma espécie de especialista do local, como discute Wang et al. [Wang et al. 2017].

Existem indicações de que esse tipo de mecanismo é muito importante para o Foursquare, ajudando a explicar o seu sucesso [Santos et al. 2017]. No entanto, pouco se sabe sobre onde e em quais categorias existe maior engajamento dos usuários, a fim de explicitar em qual

\footnotetext{
${ }^{1}$ https://foursquare.com.

${ }^{2}$ Um tipo de mecanismo de incentivo baseado em elementos de jogos.

${ }^{3}$ No contexto do trabalho, confirmar presença de forma virtual em determinado local.
} 
delas há maior disputa (Restaurantes, bares, parques). Assim, neste trabalho é estudado o mecanismo de incentivo baseado na conquista de prefeitura de estabelecimentos, a fim de detectar quais locais ou categorias de locais são mais disputados e tentar identificar possíveis motivos para essas disputas. Especificamente, é analisada a alternância de prefeitos em estabelecimentos de diversas categorias em Curitiba, Brasil, e em Chicago, EUA, pois são cidades de estudo de um projeto maior ao qual este trabalhos faz parte.

Através da interação dos usuários com o sistema foi possível analisar o mecanismo de incentivo alvo e detectar a utilidade para motivar as pessoas a realizarem mais check-ins. Assim, permitindo identificar, por exemplo, algumas das características de locais onde ocorrem disputas mais acirradas e a relação com a categoria do local ou a sua localização. Foram encontrados indícios de que, por exemplo, empresas mundialmente conhecidas podem influenciar mais significativamente o desejo dos usuários para disputar a prefeitura dos mesmos em Curitiba. Ainda nessa cidade, diferentemente de Chicago, existe uma indicação de que locais com elevada interação de grupos de indivíduos que tendem a se conhecer favorece uma maior disputa por prefeitura. Além disso, observamos que a distribuição dos locais mais disputados tendem a estar nas áreas mais ricas das duas cidades.

O estudo desenvolvido é importante, por exemplo, para auxiliar desenvolvedores interessados no mecanismo de incentivo prefeitura na utilização em novos sistemas com o intuito de melhorar a interação com o sistema. Além disso, empresas podem explorar esse mecanismo para entender melhor o engajamento com a sua marca em várias localidades distintas, desenvolvendo estratégias adaptativas onde for necessário.

O restante do trabalho é organizado da seguinte maneira. A Seção 2, apresenta os trabalhos relacionados. Na Seção 3, são apresentados detalhes do sistema e o mecanismo de incentivo em estudo. A Seção 4 explica a metodologia utilizada no trabalho. Na Seção 5, são apresentados os resultados. Por último, a Seção 6, que apresenta as conclusões do trabalho.

\section{Trabalhos Relacionados}

Existem vários trabalhos que exploram dados de check-ins ${ }^{4}$ para um melhor entendimento do comportamento dos usuários e da dinâmica de cidades. Por exemplo os trabalhos [Quercia et al. 2014, Venerandi et al. 2015, Mueller et al. 2017, Neves et al. 2016] utilizam dados do Foursquare para capturar fenômenos importantes sobre o comportamento geral de usuários nas cidades.

Usuários possuem um papel central na geração e compartilhamento de check-ins. Sendo assim, é necessário que as LBSNs mantenham os mesmos motivados. Nessa direção, Santos et al. [Santos et al. 2017] estudaram os mecanismos de incentivo prefeitura e medalhas do Foursquare. O estudo monitorou novos usuários durante 13 semanas. Os autores observam que as prefeituras parecem ser o mecanismo mais eficaz na motivação dos usuários. Wang et al. [Wang et al. 2017] analisou a efetividade de contribuidores "word-of-mouth" (boca-aboca) juntamente com elementos de incentivo, como prefeituras. Os autores demonstram que os usuários preferem seguir dicas de pessoas que são reconhecidas pelo sistema por alguma especialidade, por exemplo medalhas, ao invés de prefeitura.

Cramer et al. [Cramer et al. 2011] estudaram os motivos de check-ins de usuários nas redes sociais. Foram analisadas as redes sociais Facebook e Twitter, já que ambas possuem integração com o Foursquare. Foram escolhidos usuários aleatórios para uma entrevista e

\footnotetext{
${ }^{4}$ Ato de disponibilizar algum conteúdo geolocalizado na rede social.
} 
análise posteriormente. Os autores detectaram que determinados locais tendem a atrair mais check-ins do que outros. Além disso, usuários podem realizar check-ins em determinados locais apenas para ajudar outros, ação similar a de um like em redes sociais como Facebook.

A pesquisa de Pontes et al. [Pontes et al. 2012] identificou que usuários com prefeitura moram próximos ao local onde são prefeitos. Além disso, foi mostrado que através dos checkins pode-se descobrir a cidade onde o usuário reside. Lin et al. [Lin et al. 2017] estudaram os check-ins por categoria de estabelecimentos temporalmente para saber quais categorias de locais são mais frequentados em quais horários do dia. Os autores demonstram que apenas algumas categorias possuem um padrão recorrente, como casas noturnas com um grande número de check-ins nos finais de semana e durante o período da noite.

No estudo de Lindqvist et al. [Lindqvist et al. 2011], os autores estudaram o motivo de pessoas compartilharem localizações e porque as definiam como públicas ou privadas. $\mathrm{O}$ método aplicado foi um questionário de 55 perguntas a 219 usuários, as perguntas abordavam a rede social Foursquare e suas funcionalidades. Os autores notaram que pessoas relataram não realizar check-in em redes de fast food pois não querem que amigos na rede saibam dessa visita.

Este trabalho se difere dos anteriores pois é estudada a influência do mecanismo de incentivo prefeitura especificamente, monitorando centenas de locais distintos de várias categorias para maior abrangência dos dados a serem analisados. Este estudo é feito ainda para duas cidades similares e distintas do ponto de vista de seus países, Curitiba e Chicago, permitindo assim uma comparação dos resultados entre diferentes culturas.

\section{Foursquare-Swarm e Seus Mecanismos de Incentivo}

As LBSNs possuem como um fator chave a exploração da informação geográfica para prover serviços e funcionalidades para os usuários. O Fourquare-Swarm ${ }^{5}$ é uma LBSN responsável por permitir que usuários disponibilizem suas localizações para amigos (check-in), bem como compartilhar e acessar conteúdos a locais disponíveis na cidade.

Para as LBSNs se manterem sustentáveis é de extrema importância favorecer que usuários estejam motivados a realizarem tarefas de interesse na aplicação [Santos et al. 2017]. Nesse contexto, mecanismos de incentivo são elementos que fazem com que usuários passem ou continuem a realizar alguma determinada tarefa no sistema. Isso pode acontecer ao receber algo em troca, por exemplo, algum tipo de reconhecimento virtual ou dinheiro. No FourquareSwarm os tipos de mecanismos de incentivo são mayorship (prefeituras), medalhas e moedas virtuais.

O Foursquare-Swarm recompensa os usuários com medalhas de acordo com check-ins realizados em locais específicos. Por exemplo, o usuário pode ganhar medalhas ao realizar check-ins em locais novos, categorias novas de locais na cidade (restaurantes, parques, aeroportos, mercados e agências bancárias) e eventos.

Outro mecanismo de incentivo é a prefeitura. O usuário considerado o prefeito de um local é aquele quem mais realizou check-ins em um local específico nos últimos 30 dias. Com esse título, o usuário pode ser visto como uma espécie de autoridade do local. Um usuário pode receber esse incentivo, ou seja, ser coroado como prefeito, em vários locais ao mesmo tempo. Além disso, pode ser o prefeito de um mesmo local várias vezes, caso perca o título de prefeito e o recupere posteriormente. A alternância de prefeitos se dá quando um usuário possui mais de um check-in de diferença do atual prefeito.

\footnotetext{
${ }^{5}$ Em 2014, o Foursquare se dividiu em duas empresas, uma delas é a LBSN Foursquare-Swarm.
} 


\section{Metodologia}

\subsection{Coleta de Dados}

Os dados que foram coletados de cada local são: Nome do local; número de check-ins realizados por usuários; categoria do local; e o nome do usuário que é o prefeito atual, a fim de melhorar a riqueza das análises.

Os coletores (web crawlers [Silva et al. 2016]) foram desenvolvidos em linguagem Python. Para obter os dados, foi necessário criar uma conta para desenvolvedores junto ao Foursquare-Swarm para a utilização da API, pois é necessária autenticação.

$\mathrm{Na}$ coleta dos locais foram utilizados os identificadores dos mesmos ${ }^{6}$, foram monitorados aproximadamente 400 locais de cada cidade. Um número maior de locais tornaria inviável a realização da coleta temporal, pois o limite diário é de 1.000 requisições. Ressalta-se que a amostragem utilizada neste trabalho possui representantes de várias categorias de locais. $\mathrm{O}$ período de coleta diária foi de 20/03/2018 até 31/05/2018 (73 dias), para ambas as cidades. Os dados foram armazenados em um banco de dados não relacional (MongoDB) para serem analisados, posteriormente.

\subsection{Estudo da Alternância de Prefeituras}

Duas métricas foram propostas para avaliar o mecanismo de incentivo: Alterações e reaparições de prefeitos. As alterações de prefeitos (Disp) definem quantas trocas ocorreram em um determinado local sem considerar os usuários. Essa disputa de prefeitura é calculada pela equação $D i s p=P * \log (N+1)$, onde $P$ é o número de troca de prefeitos do local e $N$ o total de check-ins do local no período analisado. É importante ressaltar que o Foursquare-Swarm utiliza uma janela deslizante de 30 dias para determinar o prefeito. No entanto, a variável $N$ é referente a todo o período coletado. A variável $P$ analisa o usuário que se torna prefeito, sem levar em conta o usuário, apenas o número de trocas, sendo o valor mínimo zero, caso não haja troca de prefeito no local, e no máximo a quantidade de dias coletados caso haja troca todo dia.

A quantidade de reaparições de prefeitos (Reap) demonstra a disputa entre dois ou mais usuários para conquistar o cargo. As reaparições são calculadas conforme a equação $R e a p=R * \log (N+1)$, sendo $R$ o número de reaparições de usuários como prefeitos e $N$ o total de check-ins do local de estudo no período analisado. Para que ocorra alguma reaparição, deve-se ter no mínimo duas trocas de prefeitura, pois um usuário obrigatoriamente deve perder a prefeitura e recuperá-la posteriormente para contabilizar como uma reaparição.

As duas métricas permitem analisar o impacto nas diferentes categorias, pois dependendo da combinação de altos e baixos entre os resultados será possível detectar se o local é muito ou pouco disputado.

\section{Resultados}

Esta seção apresenta os resultados de disputa e reaparição de prefeitos agrupados por categorias de locais e locais individuais, com classificação e análises dos mesmos.

\subsection{Categorias de Locais}

As Figuras 1a e $1 \mathrm{~b}$ mostram boxplots para os locais individuais das 50 categorias mais populares com relação ao número de check-ins. Como é possível observar, as categorias que mais se

\footnotetext{
${ }^{6}$ https://pt.foursquare.com/v/restaurante-madalosso/4b9bd356f964a520e12936e3
} 


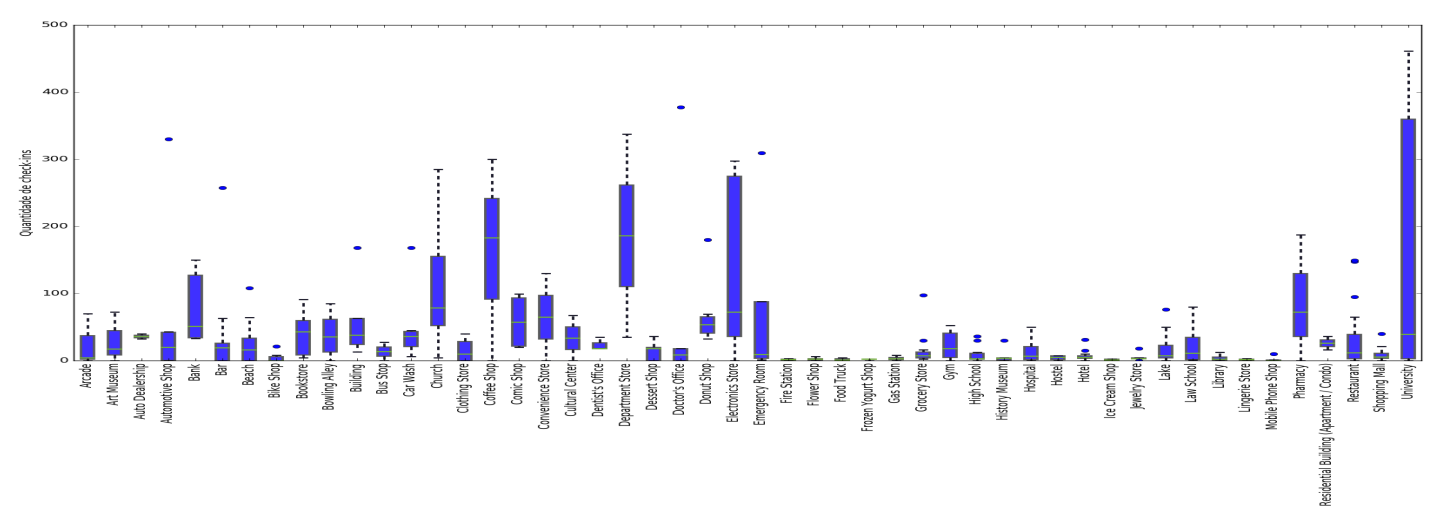

(a) Chicago

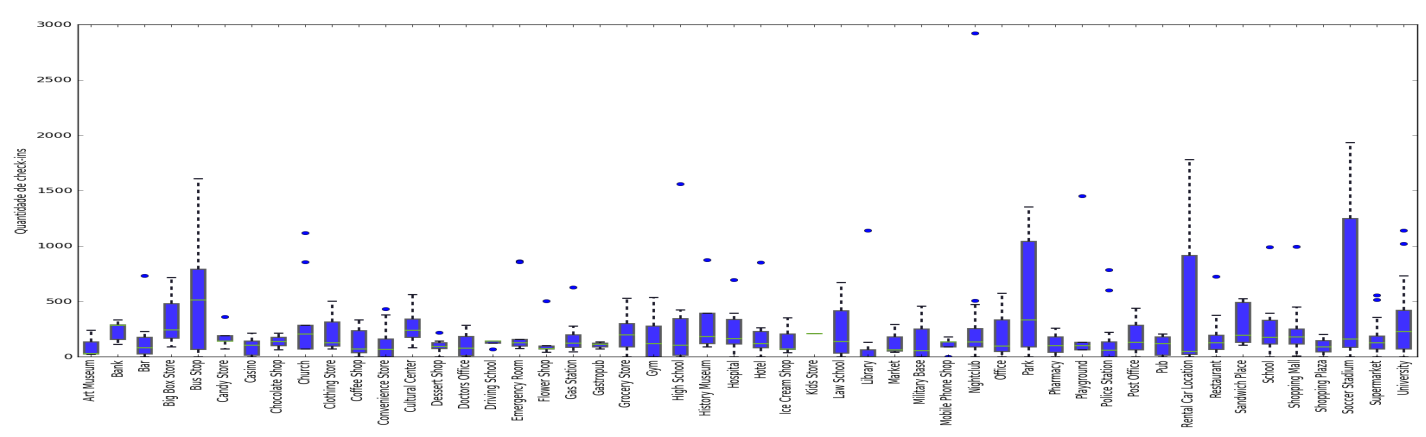

(b) Curitiba

Figura 1. Boxplot da distribuição do número de check-ins para os locais individuais das 50 categorias mais populares com relação ao número de check-ins.

destacaram em Curitiba são soccer stadium, park, rental car location e bus stop, pois a média calculada para as categorias foi acima das demais. Para a cidade de Chicago, as categorias university, eletronic store, departament store e coffee shop foram as que mais se destacaram.

Com relação às categorias com maior número de check-ins, é possível perceber diferenças das preferências em cada cidade. Por exemplo, em Chicago os check-ins foram mais comuns em estabelecimentos relacionados a comércio. Já em Curitiba estabelecimentos relacionados a lazer geram maior interesse entre os usuários para essa tarefa.

As semelhanças encontradas entre as cidades podem ser vistas nas categorias: church, universiry, restaurant e bar, pois possuem quantidade de check-ins similares para o período coletado. Com isso, é possível perceber padrões similares entre as cidades.

Com relação à disputa por categorias, as Figuras $2 \mathrm{a}$ e $2 \mathrm{~b}$ apresentam os resultados de disputas de prefeituras (Disp), agrupados por categoria. É possível observar que não existe uma relação direta entre a popularidade da categoria, em termos de número de check-ins, e o valor de disputa. Isso indica que um local ser de uma categoria popular não implica em uma maior disputa. Existem fatores implícitos que são responsáveis para ajudar a explicar esse fenômeno.

É possível observar que, em Chicago, usuários podem estar mais dispostos a disputarem por estabelecimentos relacionados a comércio, como Convenience Store. Em Curitiba usuários tendem a realizar mais disputas em locais com forte interação social de pessoas, que tendem a possuir um vínculo pessoal, como universidades, igrejas, entre outros. Esse fato pode estar associado a questões culturais, indicando que a motivação para a realização de disputa pode ser dependente da cultura local. 


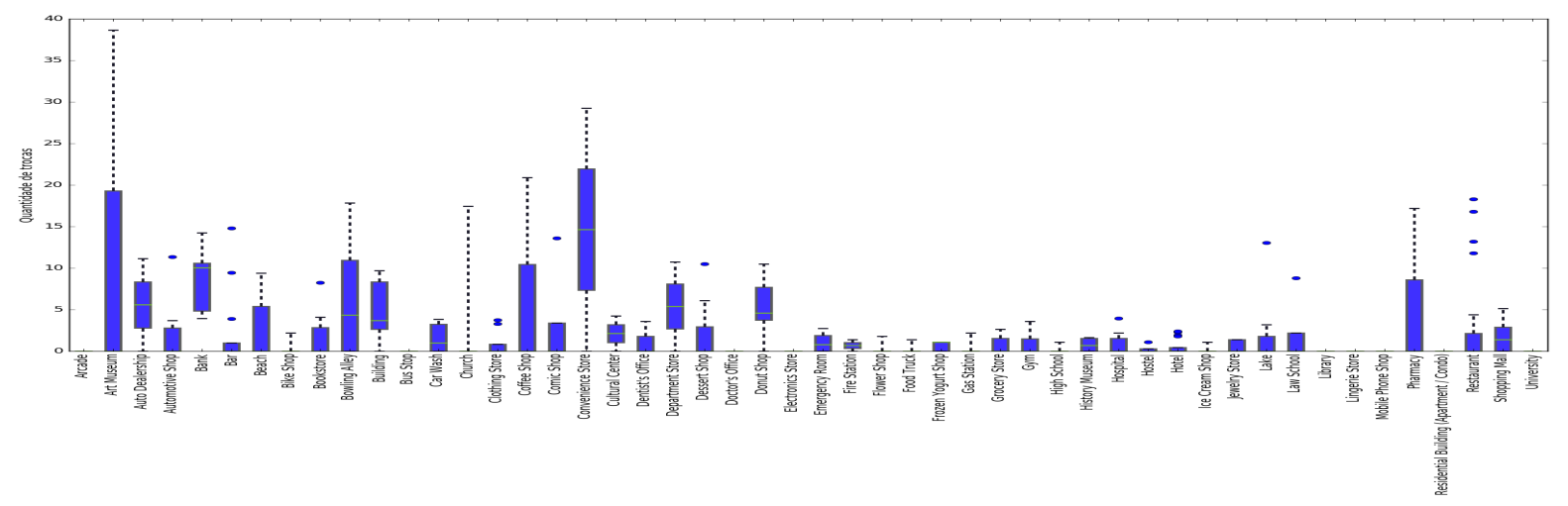

(a) Chicago

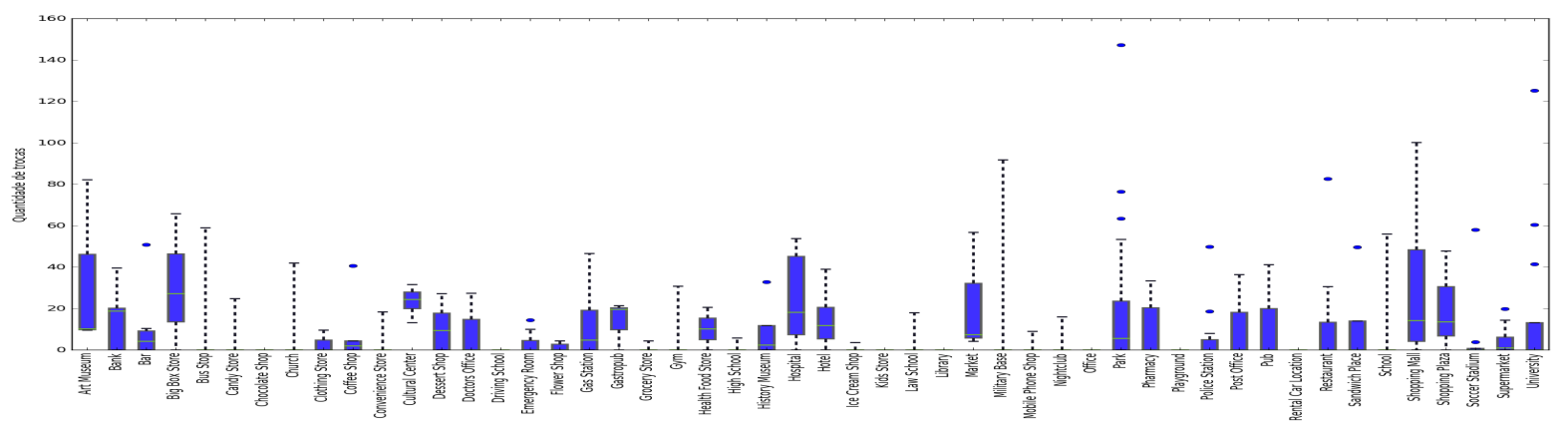

(b) Curitiba

Figura 2. Boxplot com os valores de disputa agrupados por categoria.

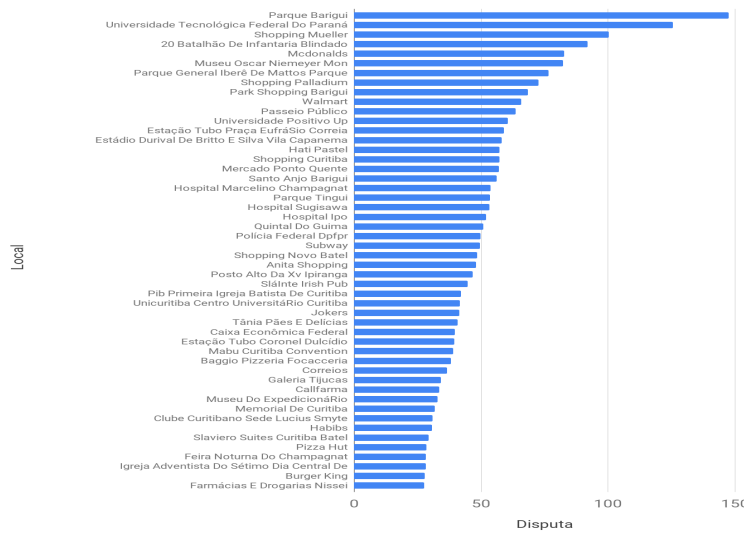

(a) Disputa em Curitiba

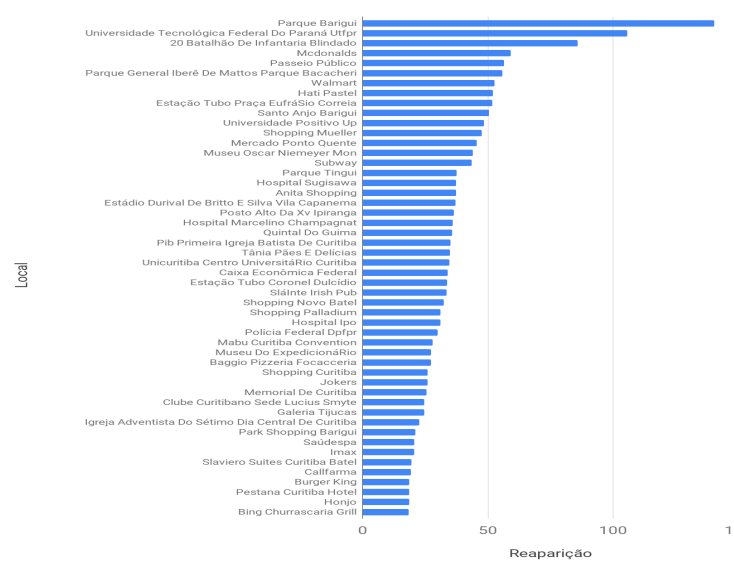

(b) Reaparição em Curitiba

Figura 3. Ranking dos 50 locais mais disputados na cidade de Curitiba.

\subsection{Locais Individuais}

O mecanismo de incentivo prefeitura consiste em favorecer disputa entre usuários pela prefeitura de locais. Assim, as trocas de prefeitos podem ser usadas para analisar se há disputa ou não no local. Caso dois usuários, ou mais, se alternem na prefeitura de um determinado local e a média de check-ins seja alta, esse local é considerado disputado. As Figuras 3 e 4 apresentam os resultados das equações de disputa e reaparição de locais individuais, conforme descrito na Seção 4.2.

Os locais individuais mais disputados entre as duas cidades são diversos. Uma con- 


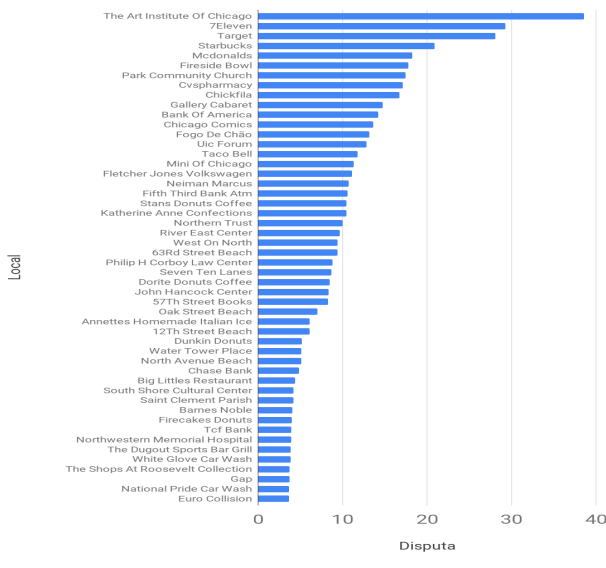

(a) Disputa em Chicago

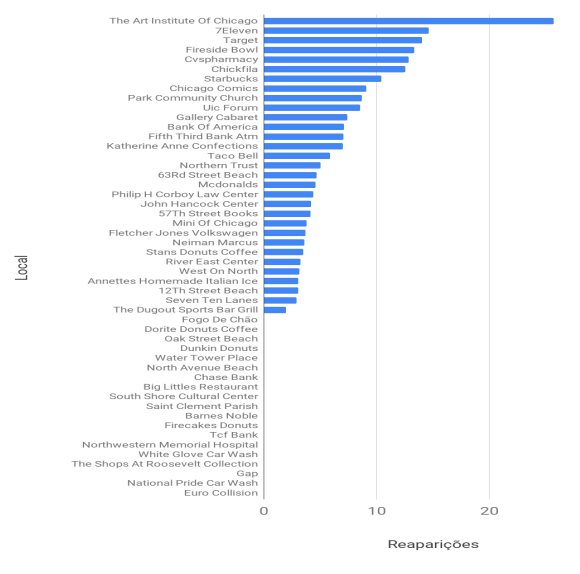

(b) Reaparição em Chicago

Figura 4. Ranking dos 50 locais mais disputados na cidade de Chicago.

cordância nos rankings não era esperada, pois, com algumas exceções, os locais individuais em cidades distintas, especialmente em outros países, tendem a ser diferentes de acordo com uma série de fatores, dentre eles fatores culturais. No entanto, é interessante notar que o estabelecimento McDonald's é disputado nas duas cidades (Figuras 3a e 4a). É interessante ainda notar que o Burger King, outra hamburgueria dos EUA, aparece entre os 50 locais mais disputados em Curitiba. É importante observar que esse tipo de dado pode ajudar empresas multinacionais, como é o caso do McDonald's e Burger King, a entender o interesse dos usuários pela empresa. Ao existir uma disputa por certos locais em detrimentos de outros da mesma categoria, existe algum fator implícito, não capturado neste trabalho, que está associado ao local. Nesse caso, usuários de Curitiba demonstram um interesse considerável em obter a prefeitura do McDonald's e Burger King, o que não é observado na mesma intensidade para outras hamburguerias da cidade.

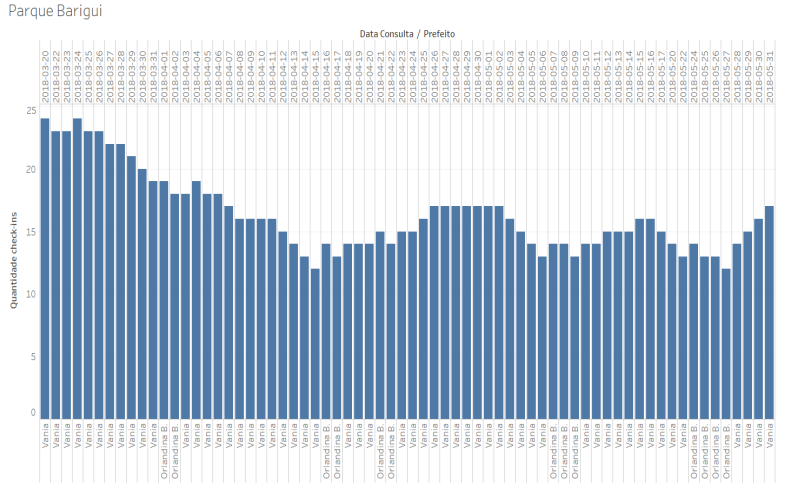

Figura 5. Disputa pela prefeitura no Parque Barigui.

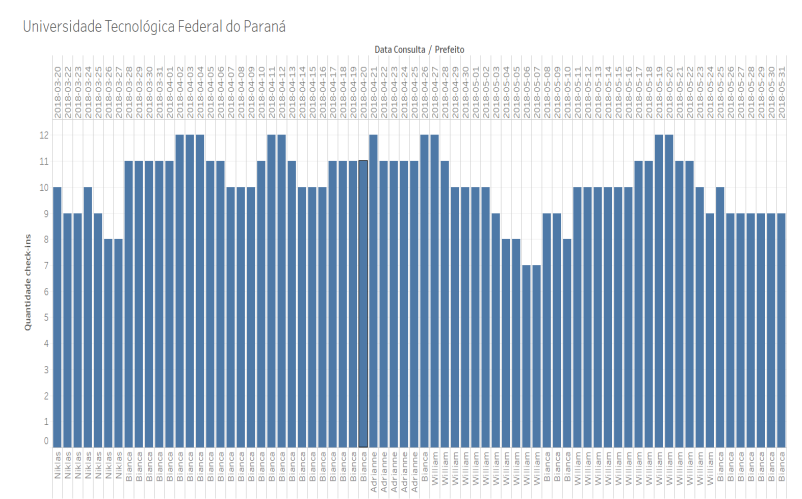

Figura 6. Disputa pela prefeitura na UTFPR.

Os resultados para a cidade de Chicago, Figura 4, indicam que disputas tendem a ser mais frequentes do que as reaparições. Muitas disputas significam que os locais tiveram vários prefeitos mas nenhum deles retornou ao cargo após a perda. Esse mesmo efeito não é observado em Curitiba, onde os prefeitos são mais engajados. Os valores da métrica de disputa e reaparições são mais altos do que os observados para Chicago.

Em Curitiba, os locais com mais reaparições são o Parque Barigui, a Universidade Tec- 
nológica Federal do Paraná (UTFPR) e o Shopping Mueller, locais de categorias distintas. Além disso, todos eles são locais de grande popularidade na cidade. Frequentar parques e bosques provavelmente faz parte da cultura dos curitibanos, isso reflete no grande número de locais dessa categoria na cidade $\left(33^{7}\right)$, tendo como grande ícone o Parque Barigui. Isso pode ajudar a explicar o interesse em obter uma prefeitura para os parques e bosques.

É interessante ainda observar que na cidade de Curitiba existem vários outros parques, universidades e shoppings, certamente, também populares na cidade. No entanto, não é possível identificar com os dados deste estudo o que torna aqueles locais em específico mais disputados. Um ponto que é possível observar é que todos os locais mais disputados citados possuem elevada interação de grupos de pessoas que tendem a se conhecer. Grupos de amigos, por exemplo, frequentam todos esses locais. Assim, ter uma prefeitura nesse tipo de local possibilita uma visibilidade que faz sentido, pois outros usuários provavelmente saberão quem é o prefeito.

Exemplos reais de disputa podem ser observados nas Figuras 5, 6 e 7 que se referem ao Parque Barigui (Curitiba), UTFPR (Curitiba) e Taco Bell (Chicago), respectivamente. Através dessas figuras é possível perceber que a disputa pela prefeitura do Parque Barigui ocorreu entre 2 usuários, que se revezaram um grande número de vezes. Apesar da média de check-ins cair um pouco com o decorrer do tempo, a predominância foi da usuária Vania, que aumentou a frequência dos check-ins ao final, possivelmente, para não perder o cargo, após ter sido interrompida algumas vezes.

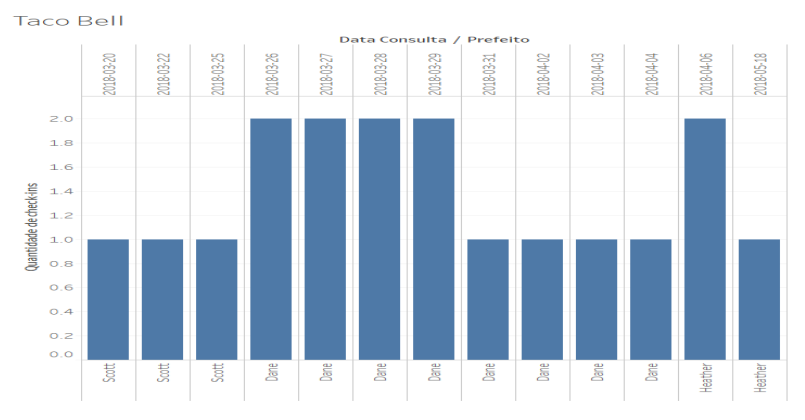

Figura 7. Disputa pela prefeitura no Taco Bell.

A quantidade de usuários que disputaram a prefeitura da UTFPR é maior se comparado com os outros locais, e a média de check-ins, apesar de ser menor que do Parque Barigui, se mantém constante. É possível perceber 7 trocas/disputas e 4 reaparições no local, com a predominância entre os usuários Bianca e William. O parque Barigui obteve um elevado valor de disputa pois na aplicação das equações consideradas, o número de check-ins (eixo Y da Figura 5), é maior do que a de outros locais. O Taco Bell é um exemplo de trocas de prefeitos sem reaparição, possui baixa quantidade de check-ins e os usuários não se motivaram a manter a prefeitura mesmo sendo fácil de adquirir o cargo, como pode ser observado na Figura 7.

A seguir são apresentados resultados referentes à dimensão espacial. A Figura 8 mostra a distribuição espacial dos 50 locais para as duas cidades com maiores valores de Disp, ou seja, locais que mais apresentaram disputa por prefeituras. Como é possível perceber nas figuras, em Curitiba a maioria dos locais é mais próximo do centro da cidade, algumas das raras exceções são, comumente, parques e shoppings. Existem locais que são localizados em áreas distantes da área central da cidade, sendo também, em alguns casos, bastante populares entre os usuários. De qualquer forma, os locais com maior número de check-ins e as categorias mais disputadas estão na região central.

\footnotetext{
${ }^{7}$ https://pt.wikipedia.org/wiki/Lista_de_parques_e_bosques_de_Curitiba.
} 


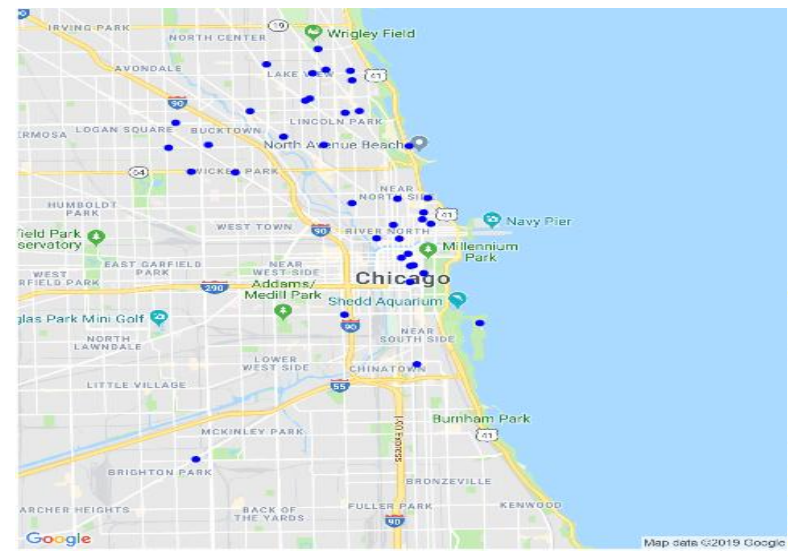

(a) Chicago

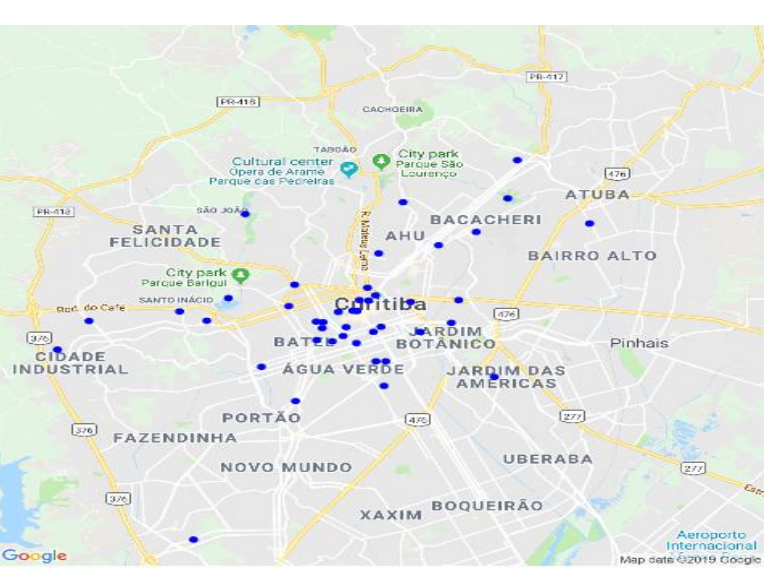

(b) Curitiba

Figura 8. Distribuição espacial dos $\mathbf{5 0}$ locais com maiores valores de $D i s p$, que leva em consideração a disputa por prefeituras de locais.

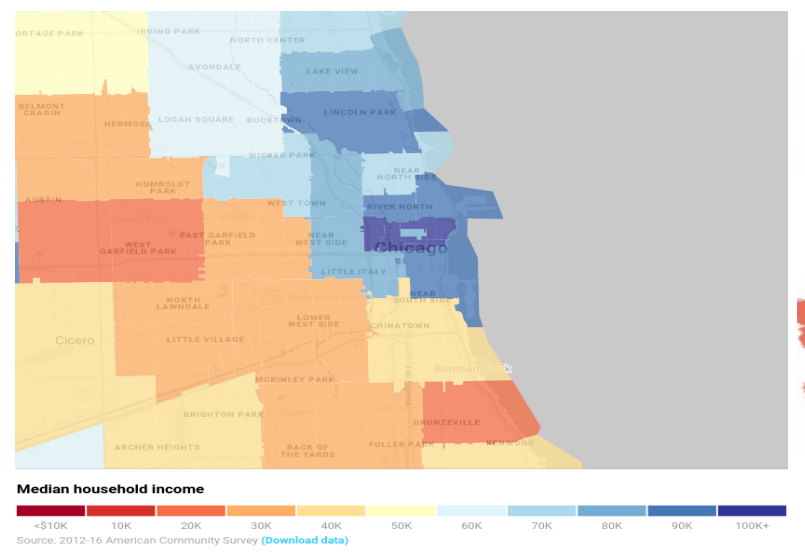

(a) Chicago

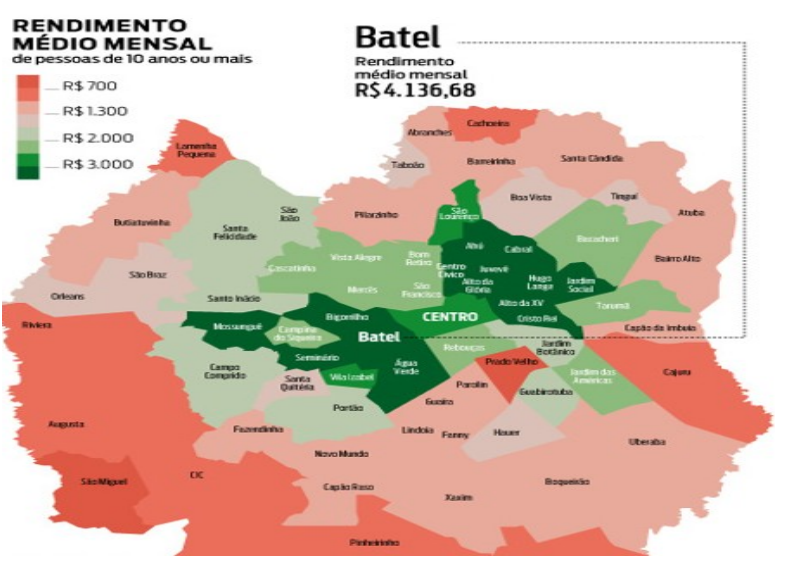

(b) Curitiba

Figura 9. Renda média por bairros.

Essa distribuição mais centralizada dos locais mais disputados não é observada na mesma proporção na cidade americana. Nessa cidade, existe uma tendência dos locais estarem distribuídos de forma mais uniformes próximos à costa e ao norte da cidade.

Foi notado que a distribuição dos locais mais disputados está nas áreas mais ricas das duas cidades. A Figura 9 mostra a renda média de bairros de Chicago (adaptado de richblockspoorblocks.com.) e Curitiba (adaptado de gazetadopovo.com.br). Esse pode ser outro fator que pode ajudar a explicar esse fenômeno, ou seja, podem ser locais que carregam implicitamente um prestígio por estarem associados a áreas nobres.

\section{Conclusão e Trabalhos Futuros}

O mecanismo de incentivo prefeitura é um mecanismo importante do Foursquare-Swarm, pois ajuda a estimular o uso do sistema. As disputas e reaparições analisadas apresentaram alguns pontos em comum entre as cidades, como no caso do McDonald's. Além disso, também foram explicitadas várias diferenças, como a concentração geográfica dos check-ins e as categorias de preferência dos usuários de cada cidade.

$\mathrm{Na}$ disputa pela prefeitura, as duas cidades apresentaram disputas em locais de categorias iguais e em categorias distintas, o que é importante para a caracterização da cidade e 
entendimento de seus indivíduos. Em ambos os casos, a distribuição dos locais mais disputados estão em áreas nobres da cidade.

Os resultados que foram apresentados para cada cidade podem estar relacionados aos costumes da população, de acordo com a alimentação, lazer e rotina, por exemplo. Foram encontrados indícios de que as motivações dos usuários para a realização de disputa são diferentes entre as duas cidades estudadas e podem ser culturalmente dependentes. Observa-se que os resultados são importantes para novos sistemas da web social que desejam implementar um mecanismo similar a fim de melhorar a interação com o usuário do mesmo, bem como para empresas que desejam analisar a aceitação de suas marcas pelos usuários, que podem estar em diferentes localidades ao redor do mundo.

O presente trabalho possui algumas possíveis limitações. A primeira é que os dados não refletem a população inteira das cidades estudadas, pois somente os usuários do software foram monitorados o que não é possível generalizar para toda a população. Outra limitação refere-se a real localização do check-in. Um usuário pode realizar um check-in em um local onde ele não está. Apesar de não existirem evidências que comprovem que isso comprometa os dados, um estudo para validar essa questão está fora do escopo deste trabalho.

Em trabalhos futuros, pretende-se analisar mais cidades com características em comum para determinar possíveis semelhanças e diferenças. Além disso, outras fontes de dados serão consideradas.

\section{Referências}

Cramer, H., Rost, M., and Holmquist, L. E. (2011). Performing a check-in: Emerging practices, norms and 'conflicts' in location-sharing using foursquare. In Proc. of MobileHCI '11, pages 57-66, New York, NY, USA. ACM.

Lin, S., Xie, R., Xie, Q., Zhao, H., and Chen, Y. (2017). Understanding user activity patterns of the swarm app: A data-driven study. In Proc. of UbiComp '17, pages 125-128, New York, NY, USA. ACM.

Lindqvist, J., Cranshaw, J., Wiese, J., Hong, J., and Zimmerman, J. (2011). I'm the mayor of my house: Examining why people use foursquare - a social-driven location sharing application. In Proc. of CHI'11, pages 2409-2418, New York, NY, USA. ACM.

Mueller, W., Silva, T. H., Almeida, J. M., and Loureiro, A. A. (2017). Gender matters! analyzing global cultural gender preferences for venues using social sensing. EPJ Data Science, 6(1):5.

Neves, Y. C. B., Sindeaux, M. P., Souza, W., Kozievitch, N. P., Loureiro, A. A., and Silva, T. H. (2016). Study of google popularity times series for commercial establishments of curitiba and chicago. In Proc. of Webmedia '16, pages 303-310, New York, NY, USA. ACM.

Pontes, T., Vasconcelos, M., Almeida, J., Kumaraguru, P., and Almeida, V. (2012). We know where you live: Privacy characterization of foursquare behavior. In Proc. of UbiComp '12 (Workshops), pages 898-905, Pittsburgh, Pennsylvania. ACM.

Quercia, D., Schifanella, R., and Aiello, L. M. (2014). The shortest path to happiness: Recommending beautiful, quiet, and happy routes in the city. In Proc. of $H T^{\prime}$ '14, pages 116-125, New York, NY, USA. ACM.

Santos, F. A., Silva, T. H., Braun, T., Loureiro, A. A. F., and Villas, L. A. (2017). Towards a sustainable people-centric sensing. In Proc. of ICC, pages $1-6$

Silva, T. H., Celes, C., andVincius Mota, J., Cunha, F., Ferreira, A., Ribeiro, A., de Melo, P., Almeida, J., and Loureiro, A. (2016). Users in the urban sensing process: challenges and research opportunities. Pervasive Computing: Next Generation Platforms for Intelligent Data Collection, Academic Press, pages 45-95.

Venerandi, A., Quattrone, G., Capra, L., Quercia, D., and Saez-Trumper, D. (2015). Measuring urban deprivation from user generated content. In Proc. of CSCW'15, pages 254-264, Vancouver, Canada. ACM.

Wang, L., Gunasti, K., Gopal, R., Shankar, R., and Pancras, J. (2017). The impact of gamification on word-of-mouth effectiveness: Evidence from foursquare. In Proc. of HICSS, Waikoloa Beach, EUA. 